\title{
Structure-Activity Relationships and structural design optimization of a series of $p$-hydroxycinnamic acids-based bis- and trisphenols as novel sustainable antiradical/antioxidant additives
}

\author{
Armando F. Reano, ${ }^{a, b, c}$ Julie Chérubin, ${ }^{a}$ Aurélien M. M. Peru, ${ }^{a, d, e}$ Qiao Wang, ${ }^{a}$ Tiphaine Clément,${ }^{a, f, g}$ Sandra \\ Domenek, $^{* b, c}$ Florent Allais*a,f,g \\ ${ }^{a}$ AgroParisTech, Chaire Agro-Biotechnologies Industrielles (ABI), 247 rue Paul Vaillant Couturier F-51100 Reims, France \\ ${ }^{\mathrm{b}}$ AgroParisTech, UMR 1145 GENIAL, 1 avenue des Olympiades, F-91744 Massy, France \\ 'INRA, UMR 1145 GENIAL, 1 avenue des Olympiades, F-91744 Massy, France \\ ${ }^{d}$ AgroParisTech, UMR 1318 IJPB, Route de Saint-Cyr F-78026 Versailles, France \\ e INRA, UMR 1318 IJPB, Route de Saint-Cyr F-78026 Versailles, France \\ ${ }^{f}$ AgroParisTech, UMR 782 GMPA, Avenue Lucien Brétignières F-78850 Thiverval-Grignon, France \\ ${ }^{\mathrm{g}}$ INRA, UMR 782 GMPA, Avenue Lucien Brétignières F-78850 Thiverval-Grignon, France \\ E-mail: florent.allais@agroparistech.fr, sandra.domenek@agroparistech.fr
}

\section{Electronic Supporting Information (ESI)}




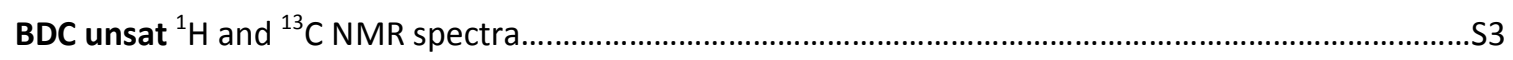

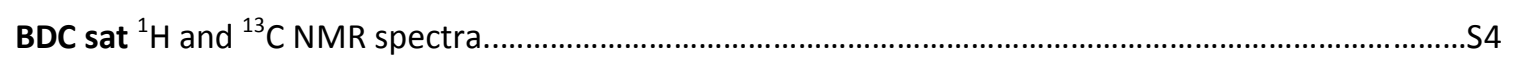

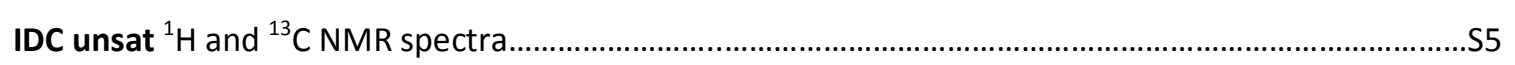

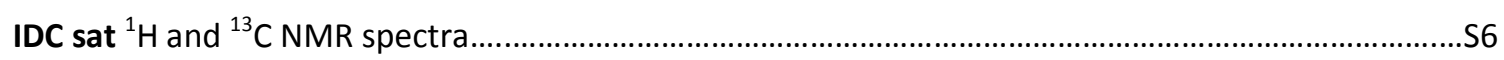

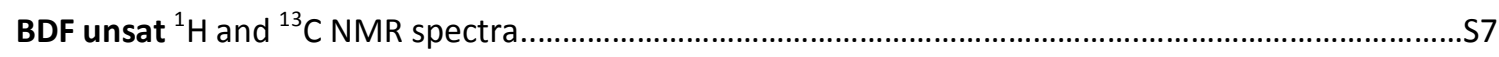

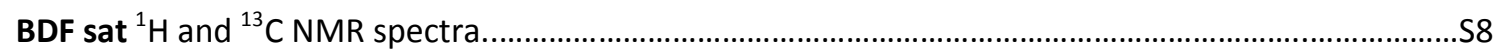

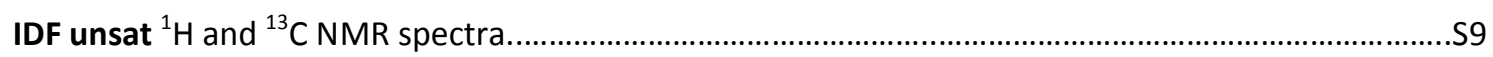

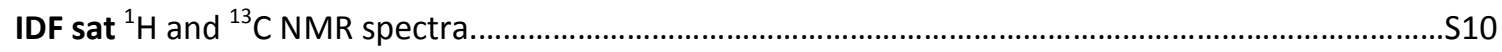

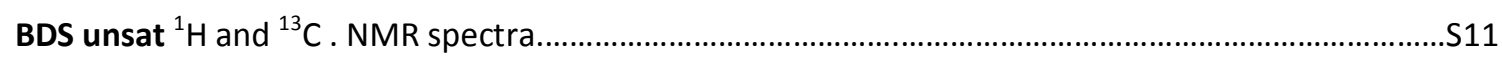

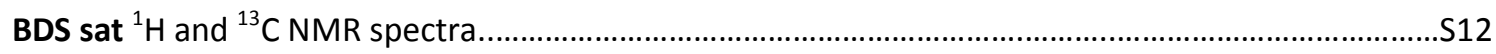

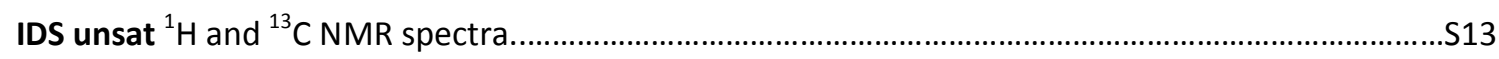

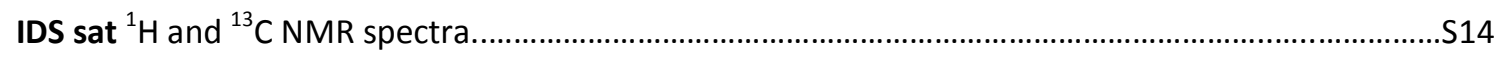

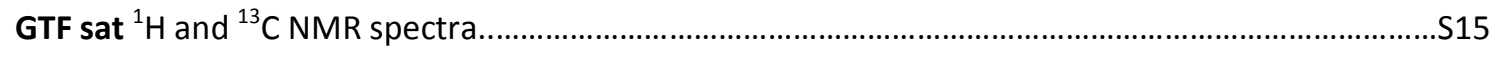

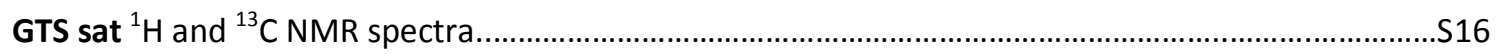




\section{BDC unsat}

White powder $\left(32 \%, \mathrm{mp} 181^{\circ} \mathrm{C}\right)$. UV: $\lambda_{\max }(\mathrm{EtOH}): 315 \mathrm{~nm}$. FTIR (neat): $v_{\max }: 1681$ (C=O), 2967 (C=C Ar), 3388 (-OH). ${ }^{1} \mathrm{H}$ NMR $\left(300 \mathrm{MHz}, \mathrm{DMSO}-d_{6}, \delta\right): 1.73\left(4 \mathrm{H}, \mathrm{m}, \mathrm{H}_{10}\right), 4.17$ (4 $\left.\mathrm{H}, \mathrm{m}, \mathrm{H}_{9}\right), 6.38\left(2 \mathrm{H}, \mathrm{d}, J=15.9, \mathrm{H}_{2}\right), 6.78(4 \mathrm{H}, \mathrm{d}, J=8.4$,

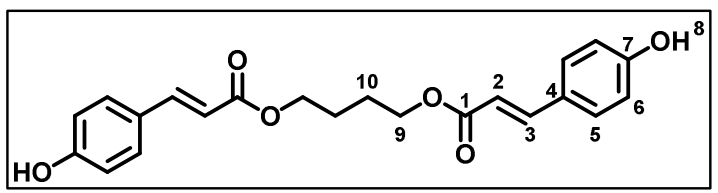

$\left.\mathrm{H}_{5}\right), 7.54\left(6 \mathrm{H}, \mathrm{m}, \mathrm{H}_{6,6,3}\right) .{ }^{13} \mathrm{C}$ NMR $\left(75 \mathrm{MHz}, \mathrm{DMSO}-\mathrm{d}_{6}, \delta\right): 25.0\left(\mathrm{t}, \mathrm{C}_{10}\right), 63.4\left(\mathrm{t}, \mathrm{C}_{9}\right), 114.1\left(\mathrm{~d}, \mathrm{C}_{2}\right), 115.7\left(\mathrm{~d}, \mathrm{C}_{5}\right)$, $125.0\left(\mathrm{~d}, \mathrm{C}_{3}\right), 130.4\left(\mathrm{~d}, \mathrm{C}_{6}\right), 144.8\left(\mathrm{~s}, \mathrm{C}_{4}\right), 159.9$ (s, $\left.\mathrm{C}_{7}\right), 166.7$ (s, C 1 ). HRMS (TOF MS, ES+): m/z calcd for $\mathrm{C}_{22} \mathrm{H}_{22} \mathrm{O}_{6} \mathrm{Na}[\mathrm{M}+\mathrm{Na}]$ : 405.1317; found: 405.1314 .

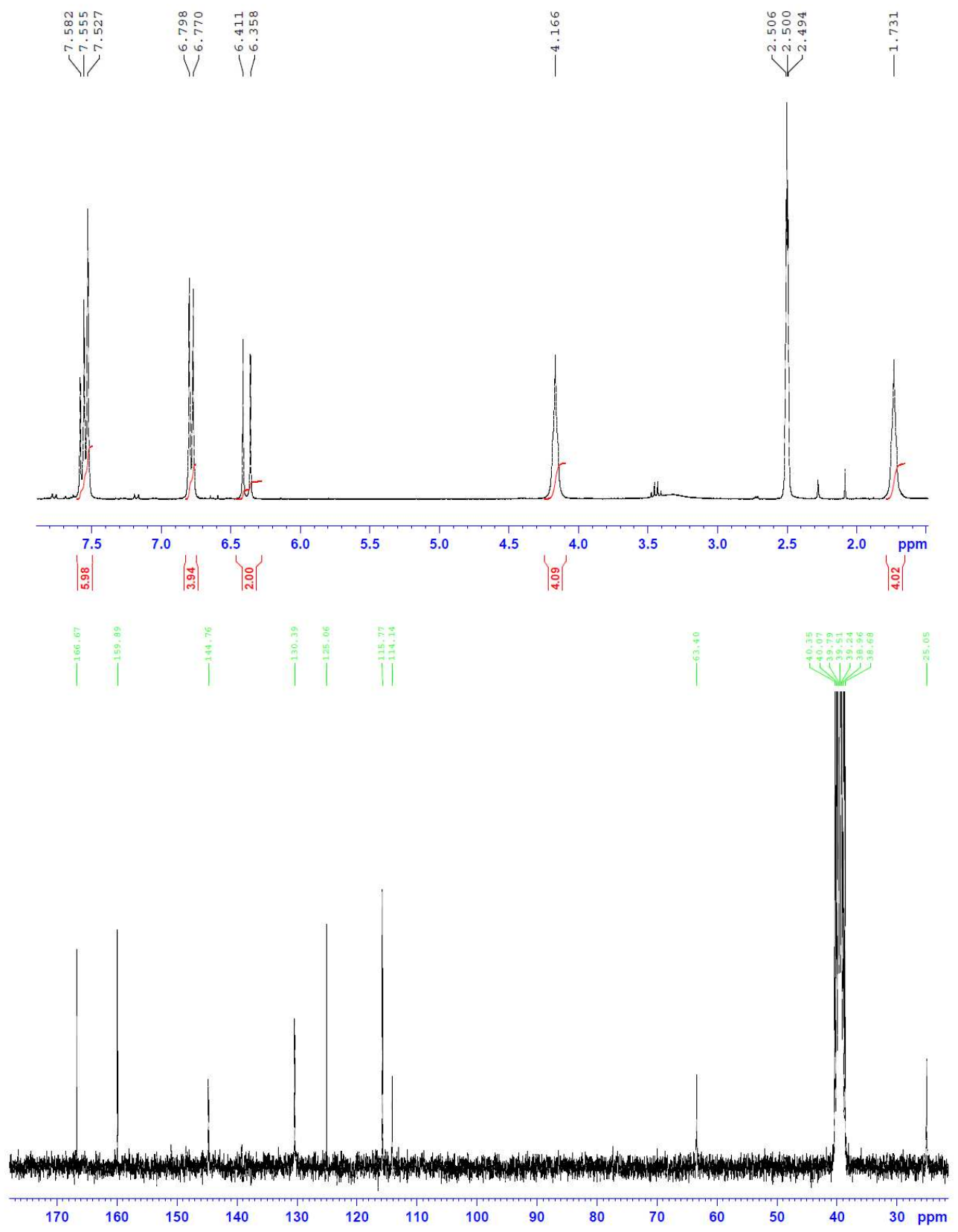




\section{BDC sat}

White powder $\left(31 \%, \mathrm{mp} 97^{\circ} \mathrm{C}\right)$. UV: $\lambda_{\max }(\mathrm{EtOH}): 205,225,280$ nm. FTIR (neat): $v_{\max }: 1712(\mathrm{C}=\mathrm{O}), 2880(\mathrm{C}=\mathrm{C} \mathrm{Ar}), 3411(-\mathrm{OH}) .{ }^{1} \mathrm{H}$ NMR (300 MHz, DMSO- $\left.d_{6}, \delta\right): 1.51\left(4 \mathrm{H}, \mathrm{m}, \mathrm{H}_{10}\right), 2.53(4 \mathrm{H}, \mathrm{m}$, $\left.\mathrm{H}_{2}\right), 2.72\left(4 \mathrm{H}, \mathrm{t}, J=7.5, \mathrm{H}_{3}\right), 3.97\left(4 \mathrm{H}, \mathrm{m}, \mathrm{H}_{9}\right), 6.65(4 \mathrm{H}, \mathrm{d}, J=8.5$

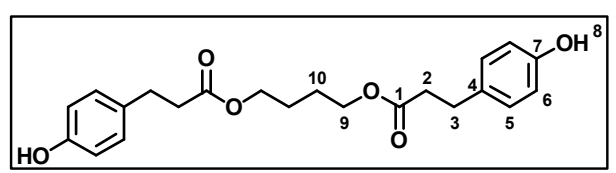
$\left.\mathrm{H}_{5}\right), 6.99\left(4 \mathrm{H}, \mathrm{d}, \mathrm{J}=8.4, \mathrm{H}_{6}\right), 9.20\left(2 \mathrm{H}, \mathrm{s}, \mathrm{H}_{8}\right) .{ }^{13} \mathrm{C} \mathrm{NMR}\left(75 \mathrm{MHz}, \mathrm{DMSO}-d_{6}, \delta\right): 24.8\left(\mathrm{t}, \mathrm{C}_{10}\right), 29.6\left(\mathrm{t}, \mathrm{C}_{2}\right), 35.6(\mathrm{t}$, $\mathrm{C}_{3}$ ), $63.3\left(\mathrm{t}, \mathrm{C}_{9}\right), 115.1\left(\mathrm{~d}, \mathrm{C}_{5}\right), 129.1\left(\mathrm{~d}, \mathrm{C}_{6}\right), 130.5\left(\mathrm{~s}, \mathrm{C}_{4}\right), 155.6\left(\mathrm{~s}, \mathrm{C}_{7}\right), 172.3\left(\mathrm{~s}, \mathrm{C}_{1}\right)$. HRMS (TOF MS, ES+): m/z calcd for $\mathrm{C}_{22} \mathrm{H}_{26} \mathrm{O}_{6} \mathrm{Na}[\mathrm{M}+\mathrm{Na}$ ]: 409.1620; found: 409.1627.
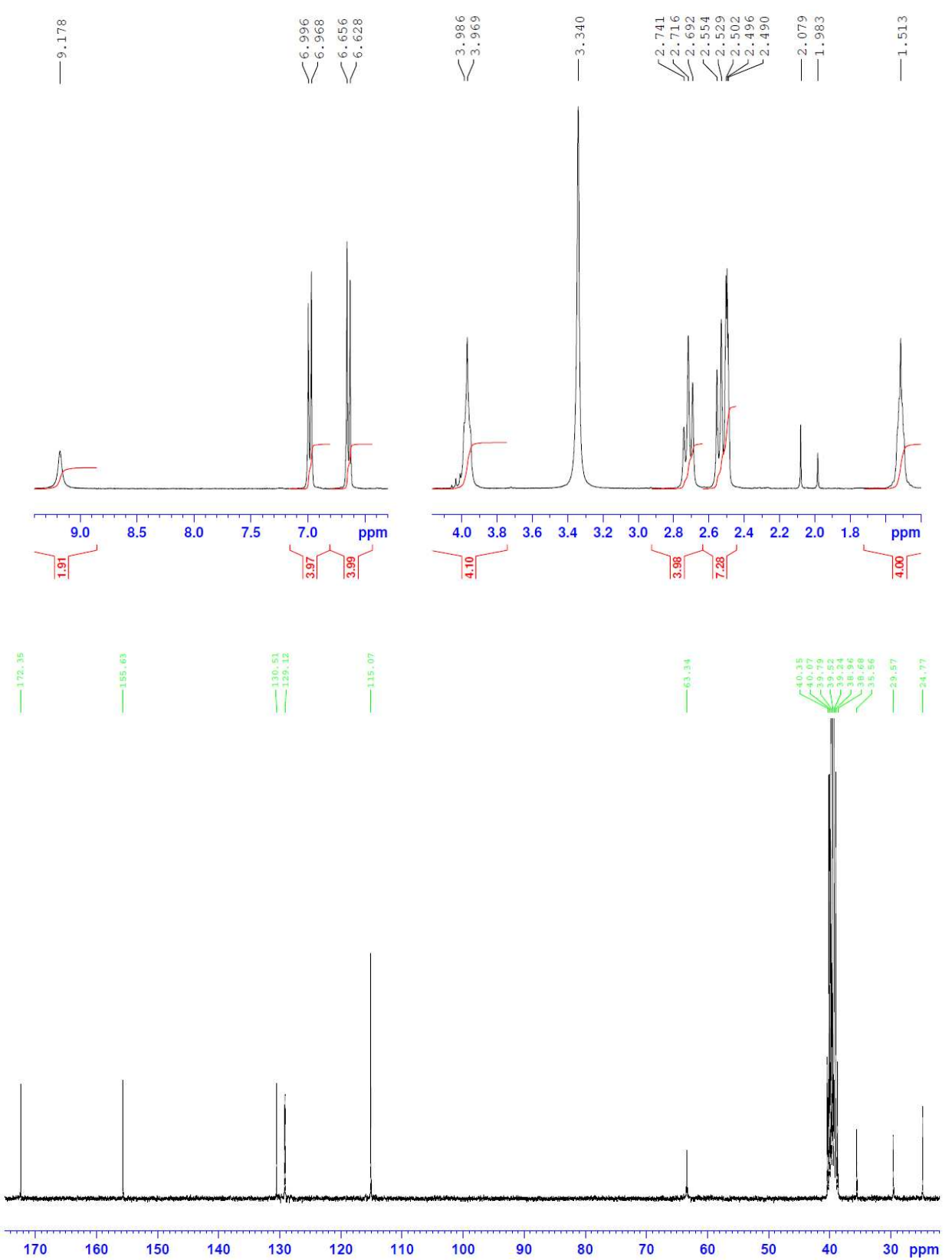


\section{IDC unsat}

White powder (36\%, mp $\left.74{ }^{\circ} \mathrm{C}\right)$. UV: $\lambda_{\max }(\mathrm{EtOH}): 230,315 \mathrm{~nm}$. FTIR (neat): $v_{\max }: 1686(\mathrm{C}=0), 3335(-\mathrm{OH}) .{ }^{1} \mathrm{H}$ NMR $\left(300 \mathrm{MHz}, \mathrm{DMSO}-d_{6}, \delta\right)$ : 3.79-3.93 $\left(4 \mathrm{H}, \mathrm{m}, \mathrm{H}_{10,10^{\prime}}\right), 4.49\left(1 \mathrm{H}, \mathrm{d}, \mathrm{J}=4.5, \mathrm{H}_{11^{\prime}}\right), 4.86\left(1 \mathrm{H}, \mathrm{t}_{\text {app }}, \mathrm{J}=5.2\right.$, $\left.\mathrm{H}_{11}\right), 5.20\left(2 \mathrm{H}, \mathrm{m}, \mathrm{H}_{9,9^{\prime}}\right), 6.41\left(2 \mathrm{H}, \mathrm{m}, \mathrm{H}_{2,2^{\prime}}\right), 6.78\left(4 \mathrm{H}, \mathrm{m}, \mathrm{H}_{5,5^{\prime}}\right), 7.59(6 \mathrm{H}$, $\left.\mathrm{m}, \mathrm{H}_{3,3^{\prime}, 6,6^{\prime}}\right), 10.0\left(2 \mathrm{H}, \mathrm{s}, \mathrm{H}_{8,8^{\prime}}\right) .{ }^{13} \mathrm{C}$ NMR $\left(75 \mathrm{MHz}, \mathrm{DMSO}-d_{6}, \delta\right): 70.3(\mathrm{t}$, $\left.\mathrm{C}_{10^{\prime}}\right), 73.1\left(\mathrm{t}, \mathrm{C}_{10}\right), 77.5\left(\mathrm{~d}, \mathrm{C}_{9,9^{\prime}}\right), 80.7\left(\mathrm{~d}, \mathrm{C}_{11^{\prime}}\right), 85.6\left(\mathrm{~d}, \mathrm{C}_{11}\right), 113.5\left(\mathrm{~d}, \mathrm{C}_{2,2^{\prime}}\right)$,

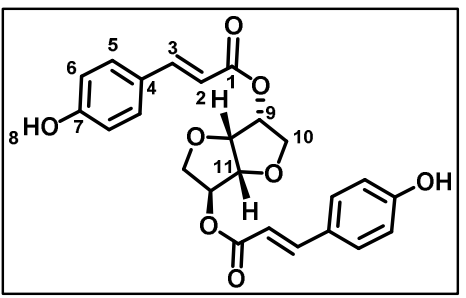
$115.8\left(\mathrm{~d}, \mathrm{C}_{5^{\prime}, 5}\right), 124.9$ (d, $\left.\mathrm{C}_{6,6^{\prime}}\right), 130.6\left(\mathrm{~d}, \mathrm{C}_{3^{\prime}}\right), 132.8\left(\mathrm{~d}, \mathrm{C}_{3}\right), 145.3\left(\mathrm{~s}, \mathrm{C}_{4^{\prime}}\right), 145.7$ (s, $\left.\mathrm{C}_{4}\right), 160.0\left(\mathrm{~s}, \mathrm{C}_{7,7^{\prime}}\right), 165.9$ (s, $\mathrm{C}_{1,1^{\prime}}$ ). HRMS (TOF MS, ES+): $\mathrm{m} / z$ calcd for $\mathrm{C}_{24} \mathrm{H}_{22} \mathrm{O}_{8} \mathrm{Na}[\mathrm{M}+\mathrm{Na}$ ]: 461.1223 ; found: 461.1212 .

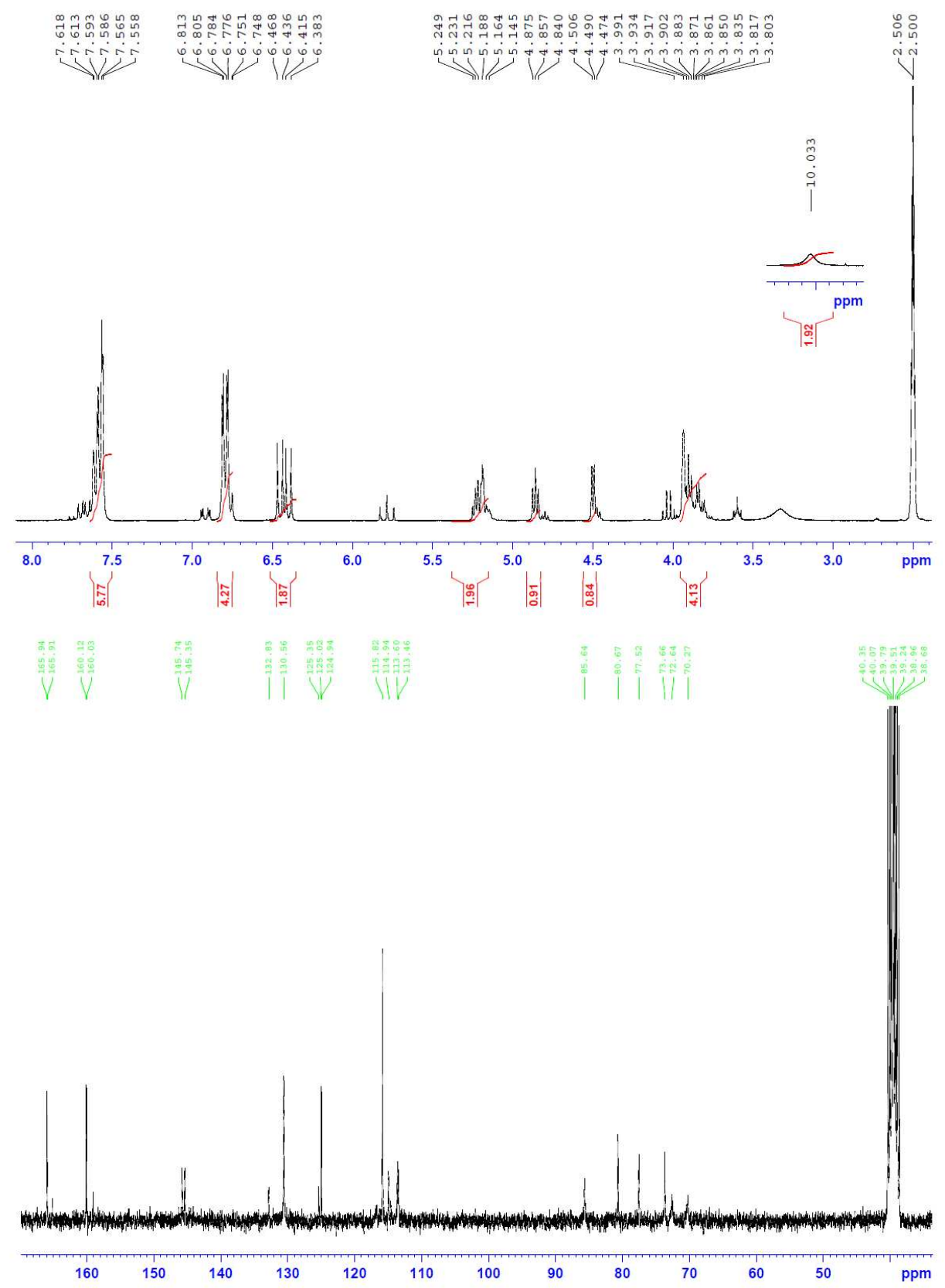




\section{IDC sat}

White powder $\left(31 \%, \mathrm{mp} 121{ }^{\circ} \mathrm{C}\right)$. UV: $\lambda_{\max }(\mathrm{EtOH}): 205,225,280 \mathrm{~nm}$. FTIR (neat): $v_{\max }: 1732(\mathrm{C}=\mathrm{O}), 2903(\mathrm{C}=\mathrm{C} \mathrm{Ar}), 3430(-\mathrm{OH}) .{ }^{1} \mathrm{H}$ NMR $(300 \mathrm{MHz}$, Acetone- $\left.d_{6}, \delta\right): 2.6\left(4 \mathrm{H}, \mathrm{m}, \mathrm{H}_{2,2^{\prime}}\right), 2.83\left(4 \mathrm{H}, \mathrm{m}, \mathrm{H}_{3,3^{\prime}}\right), 3.83-3.90\left(4 \mathrm{H}, \mathrm{m}, \mathrm{H}_{10,10^{\prime}}\right)$, $4.34\left(1 \mathrm{H}, \mathrm{d}_{a p p}, J=4.8, \mathrm{H}_{11^{\prime}}\right), 4.76\left(1 \mathrm{H}, \mathrm{t}_{a p p}, J=5.2, \mathrm{H}_{11}\right), 5.12\left(2 \mathrm{H}, \mathrm{m}, \mathrm{H}_{9,9^{\prime}}\right), 6.75$ $\left(4 \mathrm{H}, \mathrm{m}, \mathrm{H}_{5,5^{\prime}}\right), 7.06\left(4 \mathrm{H}, \mathrm{m}, \mathrm{H}_{6,6^{\prime}}\right), 8.1\left(2 \mathrm{H}, \mathrm{s}, \mathrm{H}_{8,8^{\prime}}\right) .{ }^{13} \mathrm{C} \mathrm{NMR}(75 \mathrm{MHz}$, Acetone$\left.d_{6}, \delta\right): 36.2\left(t, C_{2,2^{\prime}}\right), 70.9\left(t, C_{3^{\prime}}\right), 73.2\left(t, C_{3}\right), 74.5\left(t, C_{10^{\prime}}\right), 78.4\left(t, C_{10}\right), 81.3(d$,

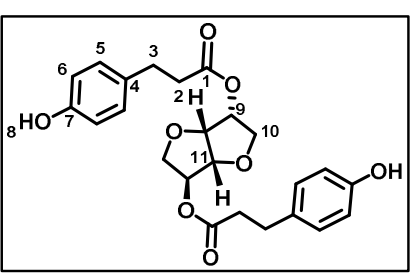
$\left.C_{9^{\prime}}\right), 86.4\left(d, C_{9}\right), 115.7\left(d, C_{5,5^{\prime}}\right), 129.8\left(d, C_{6,6^{\prime}}\right), 131.8\left(d, C_{11,11^{\prime}}\right), 156.2\left(s, C_{4,4^{\prime}}\right), 172.2\left(s, C_{7^{\prime}, 7}\right), 206.3\left(s, C_{1^{\prime}, 1}\right)$. HRMS (TOF MS, ES+): $\mathrm{m} / z$ calcd for $\mathrm{C}_{24} \mathrm{H}_{26} \mathrm{O}_{8} \mathrm{Na}$ [M + Na]: 465.1514; found: 465.1525 .

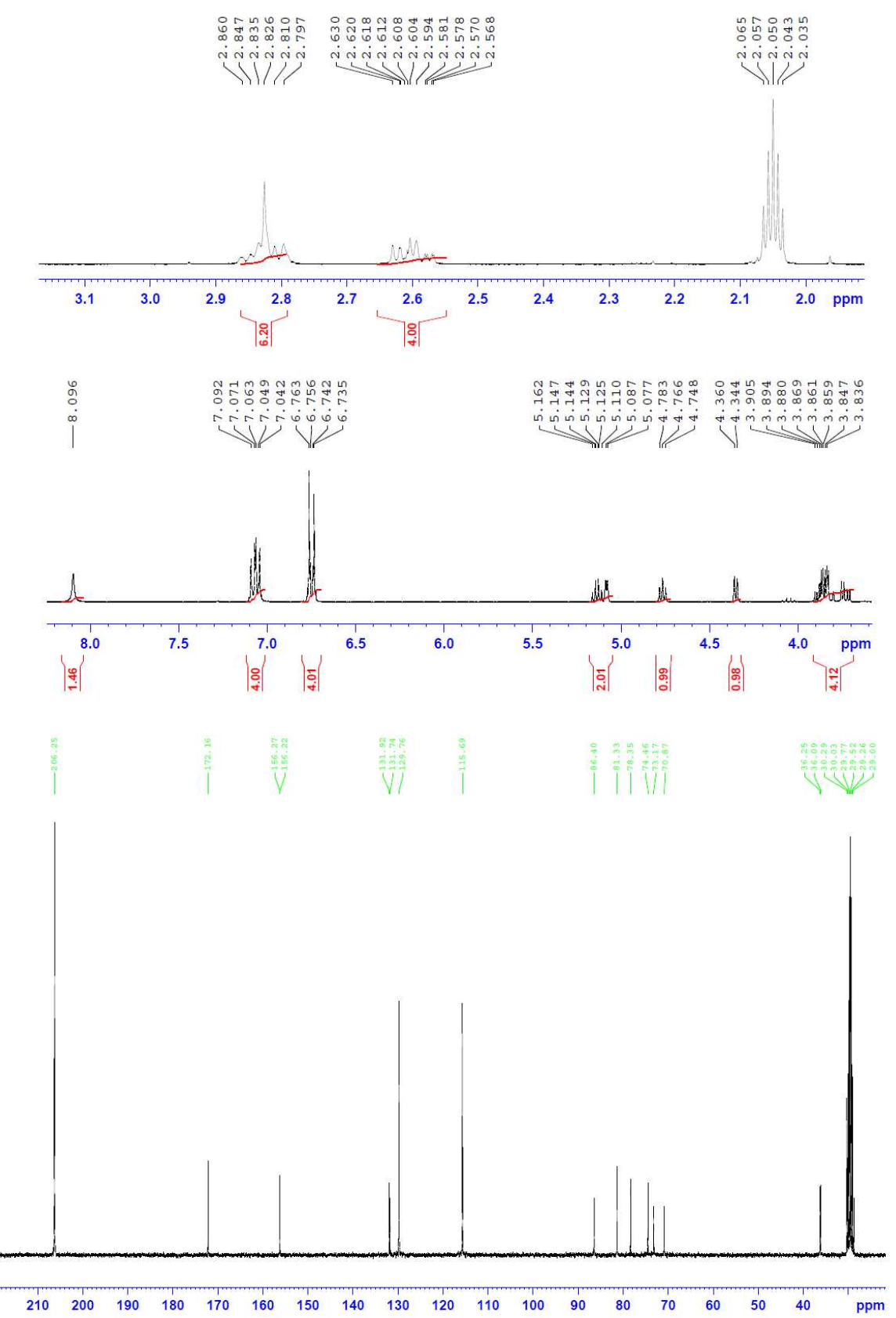




\section{BDF unsat}

White powder $\left(38 \%, \mathrm{mp} 141.7^{\circ} \mathrm{C}\right)$. UV : $\lambda_{\max }(\mathrm{EtOH})$ : 220, 240, $330 \mathrm{~nm}$. FTIR (neat): $v_{\max }: 1698$ (C=O), 2951 ( $\mathrm{C}=\mathrm{C}$ Ar), $3487(-\mathrm{OH}) .{ }^{1} \mathrm{H}$ NMR $\left(300 \mathrm{MHz}^{\mathrm{CDCl}}{ }_{3}, \delta\right): 1.75$ $\left(4 \mathrm{H}, \mathrm{m}, \mathrm{H}_{13}\right), 3.81\left(6 \mathrm{H}, \mathrm{s}, \mathrm{H}_{10}\right), 4.18\left(4 \mathrm{H}, \mathrm{m}, \mathrm{H}_{12}\right), 6.48(2$ $\left.\mathrm{H}, \mathrm{d}, J=15.9, \mathrm{H}_{2}\right), 6.79\left(2 \mathrm{H}, \mathrm{d}, J=8.1, \mathrm{H}_{8}\right), 7.11(2 \mathrm{H}, \mathrm{m}$,

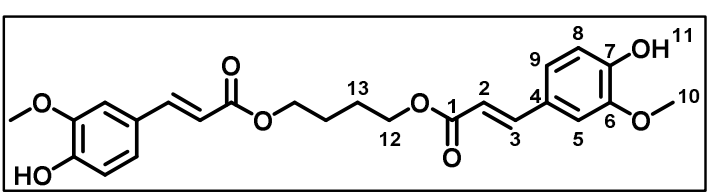
$\left.\mathrm{H}_{9}\right), 7.32\left(2 \mathrm{H}, \mathrm{m}, \mathrm{H}_{5}\right), 7.56\left(2 \mathrm{H}, \mathrm{d}, J=15.9, \mathrm{H}_{3}\right) .{ }^{13} \mathrm{C} \mathrm{NMR}\left(75 \mathrm{MHz} \mathrm{CDCl}_{3}, \delta\right): 25.5\left(\mathrm{t}, \mathrm{C}_{13}\right), 55.7\left(\mathrm{q}, \mathrm{C}_{10}\right), 63.3(\mathrm{t}$, $\left.C_{12}\right), 111.2\left(d, C_{8}\right), 114.4\left(d, C_{2}\right), 115.5\left(d, C_{9}\right), 123.2\left(d, C_{5}\right), 125.5\left(s, C_{4}\right), 145.0\left(s, C_{6}\right), 147.9\left(d, C_{3}\right), 149.4(s$, $\mathrm{C}_{7}$ ), $166.7\left(\mathrm{~s}, \mathrm{C}_{1}\right.$ ). HRMS (TOF MS, ES+): $\mathrm{m} / \mathrm{z}$ calcd for $\mathrm{C}_{24} \mathrm{H}_{26} \mathrm{O}_{8} \mathrm{Na}[\mathrm{M}+\mathrm{Na}$ ]: 465.1530; found: 465.1525.

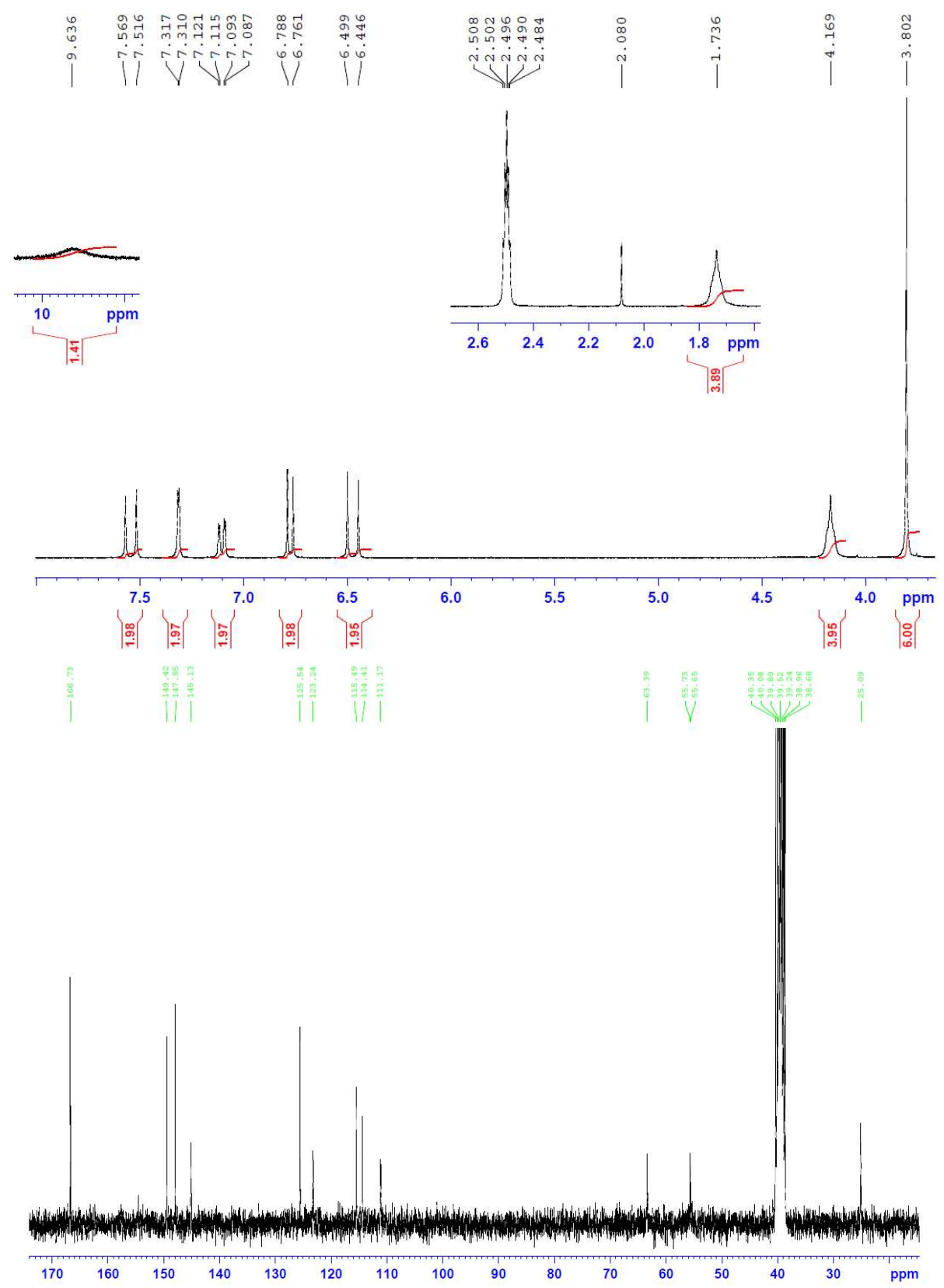




\section{BDF sat}

White powder $\left(95 \%, \mathrm{mp} 109{ }^{\circ} \mathrm{C}\right)$. UV: $\lambda_{\max }(\mathrm{EtOH}): 215$, 230, $285 \mathrm{~nm}$. FTIR (neat): $v_{\text {max }}: 1708$ (C=O), 2875 (C=C Ar), $3339(-\mathrm{OH}) .{ }^{1} \mathrm{H}$ NMR (300 MHz, $\left.\mathrm{CDCl}_{3}, \delta\right): 1.61(4 \mathrm{H}, \mathrm{m}$, $\left.\mathrm{H}_{13}\right), 2.59\left(4 \mathrm{H}, \mathrm{t}, J=7.7, \mathrm{H}_{2}\right), 2.87\left(4 \mathrm{H}, \mathrm{t}, J=7.7, \mathrm{H}_{3}\right), 3.88(6$

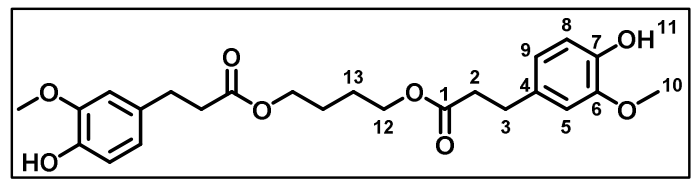
$\left.\mathrm{H}, \mathrm{s}, \mathrm{H}_{10}\right), 4.05\left(4 \mathrm{H}, \mathrm{m}, \mathrm{H}_{12}\right), 6.68\left(4 \mathrm{H}, \mathrm{m}, \mathrm{H}_{5,9}\right), 6.85\left(2 \mathrm{H}, \mathrm{m}, \mathrm{H}_{8}\right) .{ }^{13} \mathrm{C} \mathrm{NMR}\left(75 \mathrm{MHz}, \mathrm{CDCl}_{3}, \delta\right): 25.4\left(\mathrm{t}, \mathrm{C}_{13}\right)$,

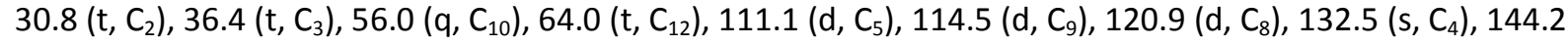
$\left(\mathrm{s}, \mathrm{C}_{6}\right), 146.5\left(\mathrm{~s}, \mathrm{C}_{7}\right), 173.1$ (s, $\mathrm{C}_{1}$ ). HRMS (TOF MS, ES+): $\mathrm{m} / z$ calcd for $\mathrm{C}_{24} \mathrm{H}_{30} \mathrm{O}_{8} \mathrm{Na}[\mathrm{M}+\mathrm{Na}$ ]: 469.1838; found: 469.1826.

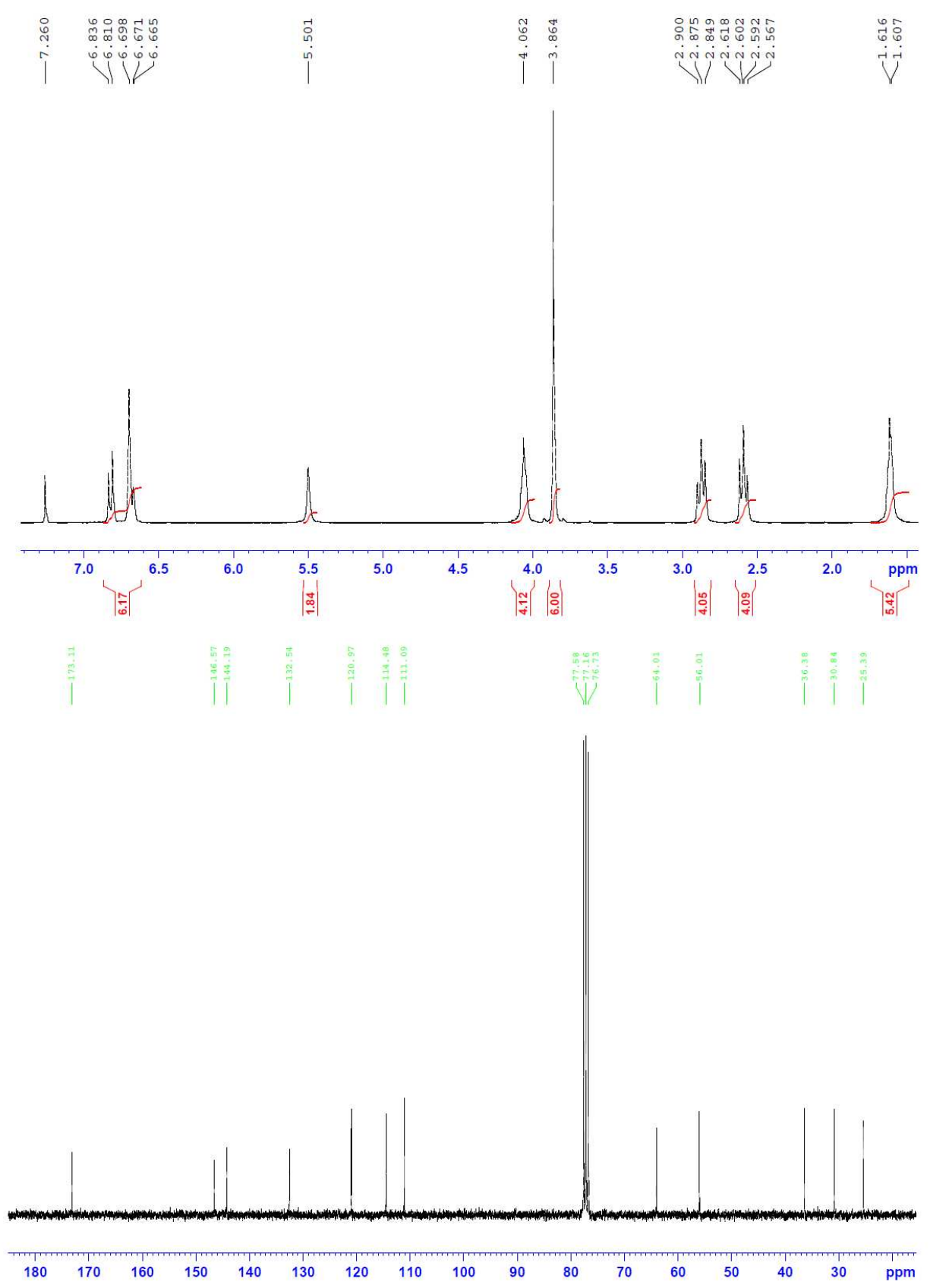




\section{IDF unsat}

White powder $\left(40 \%, \mathrm{mp} 74^{\circ} \mathrm{C}\right)$. UV: $\lambda_{\max }(\mathrm{EtOH}): 220,235,330 \mathrm{~nm}$. FTIR (neat): $v_{\text {max }}: 1700$ (C=O), 2878 (C=C Ar), $3380(-\mathrm{OH}) .{ }^{1} \mathrm{H}$ NMR (300 MHz, $\left.\mathrm{CDCl}_{3}, \delta\right)$ : 3.86-4.15 (4 H, m, $\left.\mathrm{H}_{13,13^{\prime}}\right), 3.91\left(6 \mathrm{H}, \mathrm{s}, \mathrm{H}_{10,10^{\prime}}\right), 4.61(1 \mathrm{H}, \mathrm{d}, J=4.5$, $\left.\mathrm{H}_{14^{\prime}}\right), 4.95\left(1 \mathrm{H}, \mathrm{t}_{a p p}, J=4.8, \mathrm{H}_{14}\right), 5.32\left(2 \mathrm{H}, \mathrm{m}, \mathrm{H}_{12,12^{\prime}}\right), 5.91\left(2 \mathrm{H}, \mathrm{s}, \mathrm{H}_{11,11^{\prime}}\right)$, $6.27\left(1 \mathrm{H}, \mathrm{d}, J=15.9, \mathrm{H}_{2}\right), 6.35\left(1 \mathrm{H}, \mathrm{d}, J=15.9, \mathrm{H}_{2^{\prime}}\right), 6.92\left(2 \mathrm{H}, \mathrm{m}, \mathrm{H}_{5,5^{\prime}}\right), 7.07$ (4 $\left.\mathrm{H}, \mathrm{m}, \mathrm{H}_{8^{\prime}, 8,9^{\prime}, 9}\right), 7.62\left(1 \mathrm{H}, \mathrm{d}, \mathrm{J}=15.9, \mathrm{H}_{3}\right), 6.66\left(1 \mathrm{H}, \mathrm{d}, \mathrm{J}=15.9, \mathrm{H}_{3^{\prime}}\right) .{ }^{13} \mathrm{C}$ NMR (75 $\left.\mathrm{MHz} \mathrm{CDCl}_{3}, \delta\right): 56.1\left(\mathrm{q}, \mathrm{C}_{10^{\prime}}\right), 60.5\left(\mathrm{q}, \mathrm{C}_{10}\right), 74.1\left(\mathrm{t}, \mathrm{C}_{13,13^{\prime}}\right), 78.1\left(\mathrm{~d}, \mathrm{C}_{12^{\prime}}\right), 81.2$

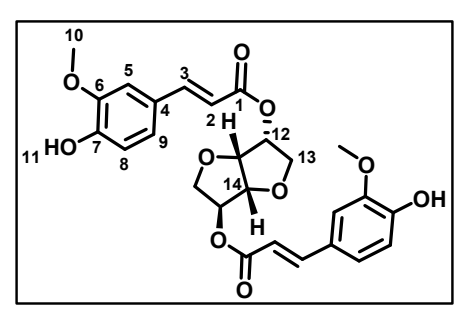
$\left(d, C_{12}\right), 86.2\left(d, C_{14,14^{\prime}}\right), 109.4\left(d, C_{2,2^{\prime}}\right), 114.6\left(d, C_{5^{\prime}}\right), 114.9\left(d, C_{5}\right), 123.5\left(d, C_{9,9^{\prime}}\right), 126.9\left(d, C_{8,8^{\prime}}\right), 146.1(d$, $\left.\mathrm{C}_{3^{\prime}, 3}\right), 146.9\left(\mathrm{~s}, \mathrm{C}_{4^{\prime}, 4}\right), 148.3\left(\mathrm{~s}, \mathrm{C}_{6,6^{\prime}}\right), 148.4\left(\mathrm{~s}, \mathrm{C}_{7,7^{\prime}}\right), 166.7$ (s, $\left.\mathrm{C}_{1,1^{\prime}}\right)$. HRMS (TOF MS, ES+): m/z calcd for $\mathrm{C}_{26} \mathrm{H}_{26} \mathrm{O}_{10} \mathrm{Na}[\mathrm{M}+\mathrm{Na}]:$ 521.1433; found: 521.1424 .

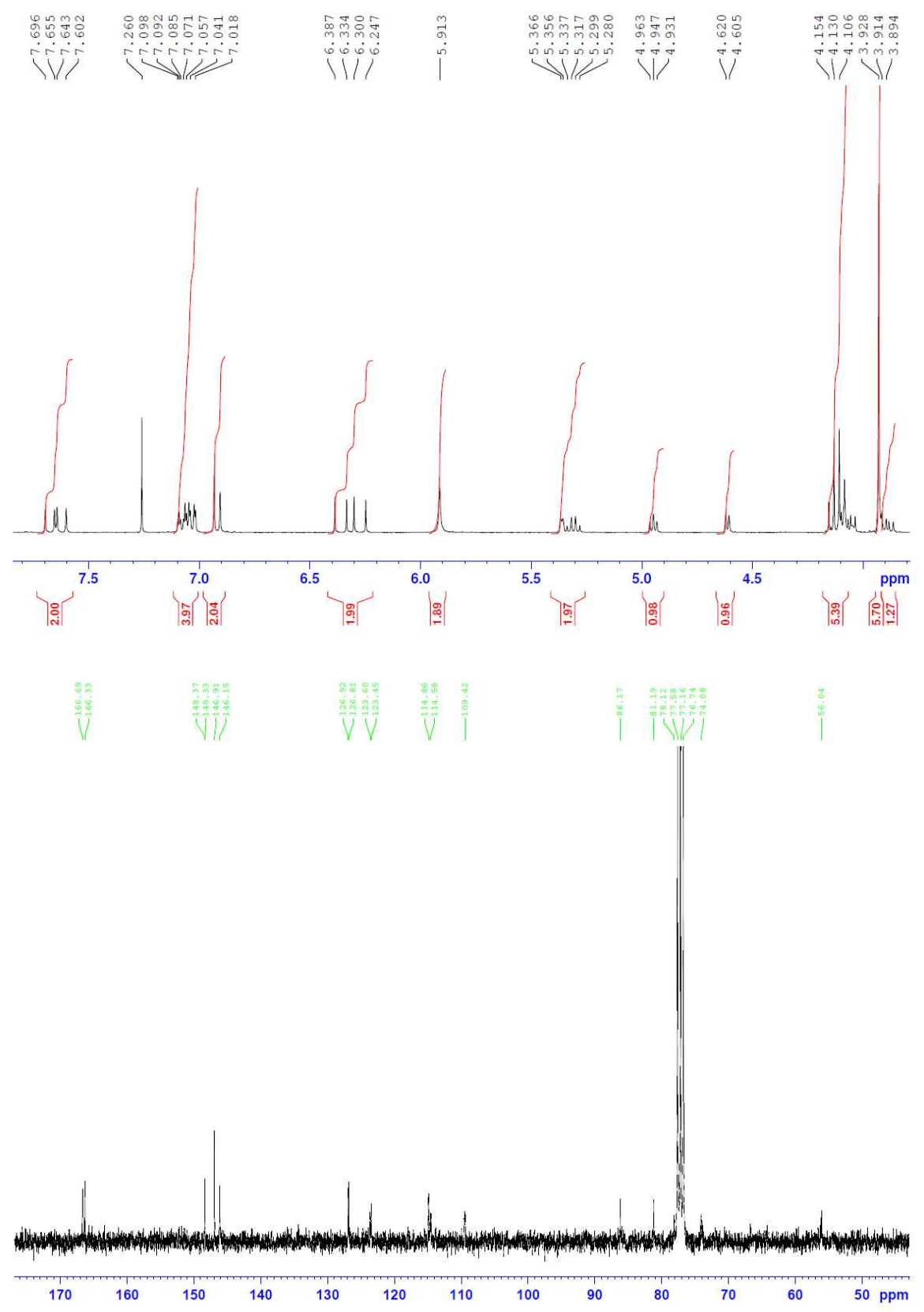




\section{IDF sat}

Yellow oil (92\%). UV: $\lambda_{\max }(\mathrm{EtOH}): 205,230,280 \mathrm{~nm}$. FTIR (neat): $v_{\max }$ : $1733(\mathrm{C}=\mathrm{O}), 2936$ ( $\mathrm{C}=\mathrm{C}$ Ar), $3400(-\mathrm{OH}) .{ }^{1} \mathrm{H} \mathrm{NMR}\left(300 \mathrm{MHz}_{\mathrm{C}} \mathrm{CDCl}_{3}, \delta\right)$ : $2.66\left(4 \mathrm{H}, \mathrm{m}, \mathrm{H}_{2,2^{\prime}}\right), 2.87\left(4 \mathrm{H}, \mathrm{m}, \mathrm{H}_{3,3^{\prime}}\right), 3.74-3.90\left(4 \mathrm{H}, \mathrm{m}, \mathrm{H}_{13,13^{\prime}}\right), 3.85$ (6 $\left.\mathrm{H}, \mathrm{s}, \mathrm{H}_{10,10^{\prime}}\right), 4.36\left(1 \mathrm{H}, \mathrm{d}, J=4.8, \mathrm{H}_{14^{\prime}}\right), 4.76\left(1 \mathrm{H}, \mathrm{t}_{\text {app }}, J=4.9, \mathrm{H}_{14}\right), 5.15(2$

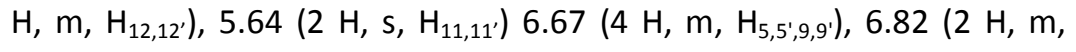
$\left.\mathrm{H}_{8,8^{\prime}}\right) .{ }^{13} \mathrm{C}$ NMR $\left(75 \mathrm{MHz}, \mathrm{CDCl}_{3}, \delta\right): 25.4\left(\mathrm{t}, \mathrm{C}_{13,13^{\prime}}\right), 30.8\left(\mathrm{t}, \mathrm{C}_{2,2^{\prime}}\right), 36.4(\mathrm{t}$, $\left.\mathrm{C}_{3,3^{\prime}}\right), 56.0\left(\mathrm{q}, \mathrm{C}_{10,10^{\prime}}\right), 64.0\left(\mathrm{~d}, \mathrm{C}_{12,12^{\prime}}\right), 111.1\left(\mathrm{~d}, \mathrm{C}_{5,5^{\prime}}\right), 114.5\left(\mathrm{~d}, \mathrm{C}_{9,9^{\prime}}\right), 120.9$

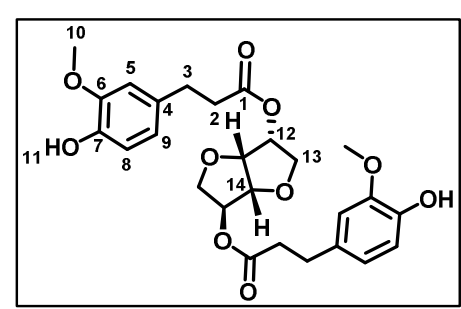
$\left(\mathrm{d}, \mathrm{C}_{8,8^{\prime}}\right), 132.5\left(\mathrm{~s}, \mathrm{C}_{4,4^{\prime}}\right), 144.2\left(\mathrm{~s}, \mathrm{C}_{6,6^{\prime}}\right), 146.5\left(\mathrm{~s}, \mathrm{C}_{7,7^{\prime}}\right), 173.1$ (s, $\left.\mathrm{C}_{1,1^{\prime}}\right)$. HRMS (TOF MS, ES+): m/z calcd for $\mathrm{C}_{26} \mathrm{H}_{30} \mathrm{O}_{10} \mathrm{Na}[\mathrm{M}+\mathrm{Na}]:$ 525.1737; found: 525.1733 .

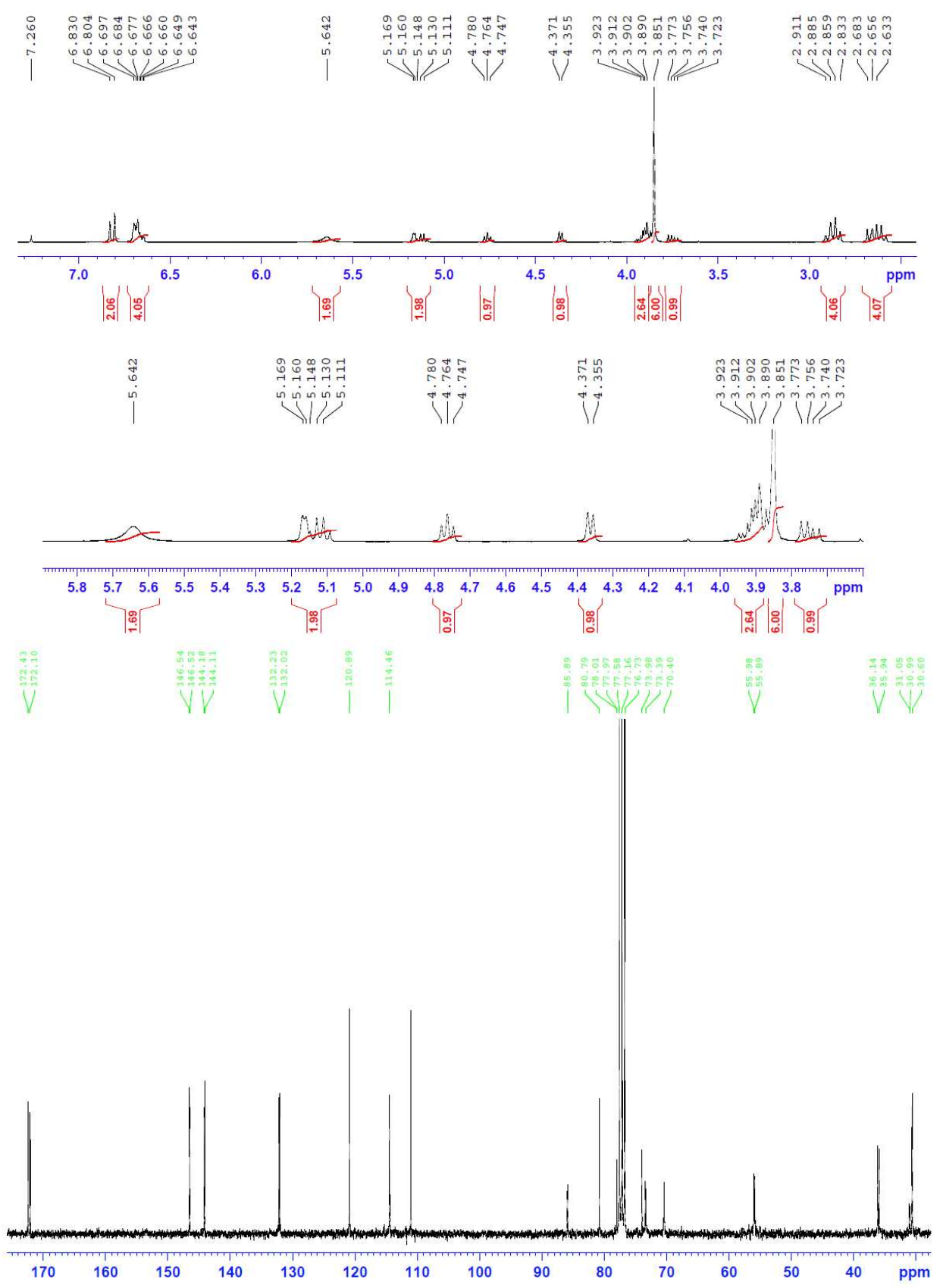




\section{BDS unsat}

White powder (31\%, mp $\left.166{ }^{\circ} \mathrm{C}\right)$. UV: $\lambda_{\max }(\mathrm{EtOH}): 328 \mathrm{~nm}$. FTIR (neat): $v_{\max }: 1695$ (C=O), 2939 (C=C Ar), $3418(-\mathrm{OH}) .{ }^{1} \mathrm{H}$ NMR $\left(300 \mathrm{MHz}, \mathrm{CDCl}_{3}, \delta\right): 1.85\left(4 \mathrm{H}, \mathrm{m}, \mathrm{H}_{11}\right), 3.9\left(12 \mathrm{H}, \mathrm{s}, \mathrm{H}_{8}\right)$, $4.26\left(4 \mathrm{H}, \mathrm{m}, \mathrm{H}_{10}\right), 5.81\left(2 \mathrm{H}, \mathrm{s}, \mathrm{H}_{9}\right), 6.26\left(2 \mathrm{H}, \mathrm{d}, \mathrm{J}=15.9, \mathrm{H}_{2}\right)$, $6.75\left(4 \mathrm{H}, \mathrm{s}, \mathrm{H}_{5}\right), 7.55\left(2 \mathrm{H}, \mathrm{d}, J=15.9, \mathrm{H}_{3}\right) .{ }^{13} \mathrm{C} \mathrm{NMR}(75 \mathrm{MHz}$,

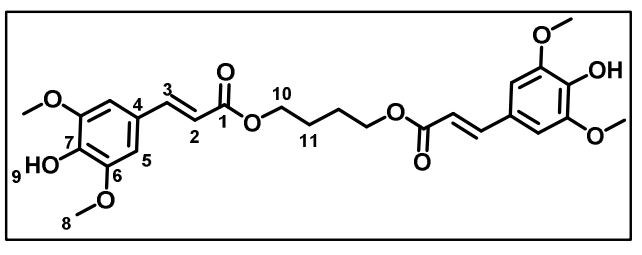
$\left.\mathrm{CDCl}_{3}, \delta\right): 25.7\left(\mathrm{t}, \mathrm{C}_{11}\right), 56.5\left(\mathrm{q}, \mathrm{C}_{8}\right), 64.1\left(\mathrm{t}, \mathrm{C}_{10}\right), 105.1\left(\mathrm{~d}, \mathrm{C}_{5}\right), 115.0\left(\mathrm{~d}, \mathrm{C}_{2}\right), 126.0\left(\mathrm{~s}, \mathrm{C}_{4}\right), 137.3\left(\mathrm{~s}, \mathrm{C}_{7}\right), 145.3$ $\left(d, C_{3}\right), 147.3\left(s, C_{6}\right), 167.3\left(s, C_{1}\right)$. HRMS (TOF MS, ES+): $m / z$ calcd for $\mathrm{C}_{26} \mathrm{H}_{30} \mathrm{O}_{10} \mathrm{Na}$ [M + Na]: 525.1740; found: 525.1737 .
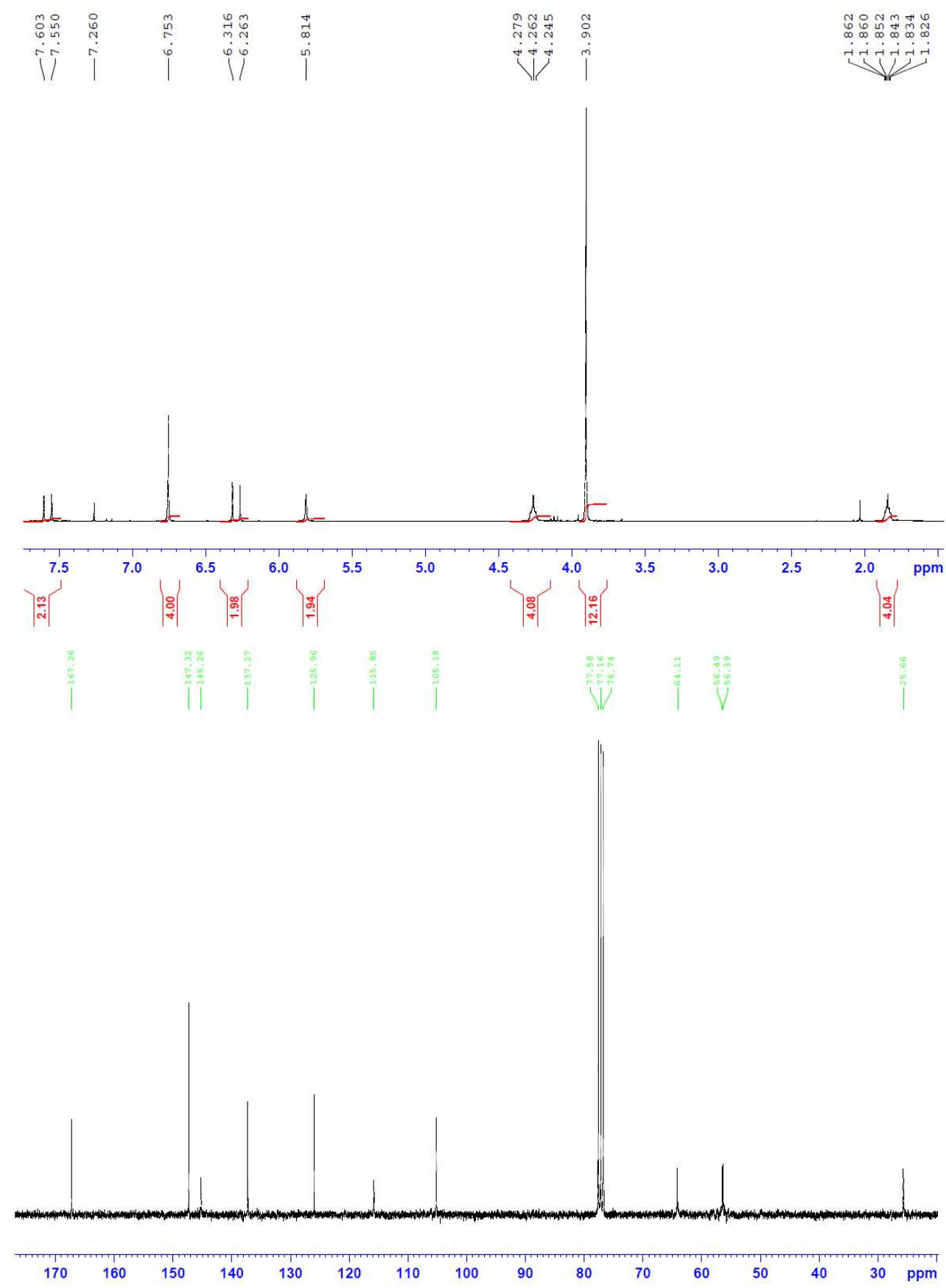


\section{BDS sat}

Powder (87\%, mp $\left.82{ }^{\circ} \mathrm{C}\right)$. UV: $\lambda_{\max }(\mathrm{EtOH}): 274 \mathrm{~nm}$. FTIR (neat): $v_{\text {max }}: 1709$ (C=O), 2933 (C=C Ar), $3372(-\mathrm{OH}) .{ }^{1} \mathrm{H}$ NMR (300 MHz, $\left.\mathrm{CDCl}_{3}, \delta\right): 1.66\left(4 \mathrm{H}, \mathrm{t}, \mathrm{H}_{11}\right), 2.6\left(4 \mathrm{H}, \mathrm{t}, J=7.7, \mathrm{H}_{2}\right), 2.88(4 \mathrm{H}, \mathrm{t}$, J=7.7, $\left.\mathrm{H}_{3}\right), 3.87\left(12 \mathrm{H}, \mathrm{s}, \mathrm{H}_{8}\right), 4.08\left(4 \mathrm{H}, \mathrm{m}, \mathrm{H}_{10}\right), 5.38\left(2 \mathrm{H}, \mathrm{s}, \mathrm{H}_{9}\right)$, $6.42\left(4 \mathrm{H}, \mathrm{s}, \mathrm{H}_{5}\right) .{ }^{13} \mathrm{C}$ NMR $\left(75 \mathrm{MHz}, \mathrm{CDCl}_{3}, \delta\right): 25.3\left(\mathrm{t}, \mathrm{C}_{14}\right), 31.3$

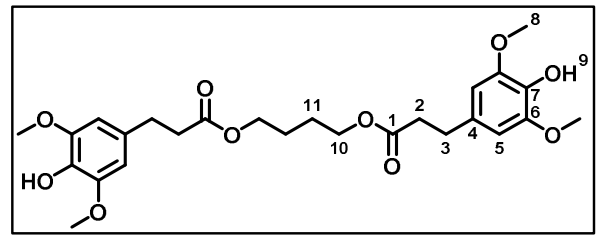

$\left(t, C_{2}\right), 36.3$ (t, $\left.C_{3}\right), 56.3$ (q, $\left.C_{10}\right), 64.0$ (t, $\left.C_{13}\right), 105.0$ (d, $\left.C_{5}\right), 131.7$ (s, $\left.C_{4}\right), 133.2$ (s, $\left.C_{7}\right), 147.1$ (s, C 6 ), 173.0 (s, $\mathrm{C}_{1}$ ). HRMS (TOF MS, ES+): $\mathrm{m} / z$ calcd for $\mathrm{C}_{26} \mathrm{H}_{34} \mathrm{O}_{10} \mathrm{Na}[\mathrm{M}+\mathrm{Na}$ ]: 529.2043; found: 529.2050.
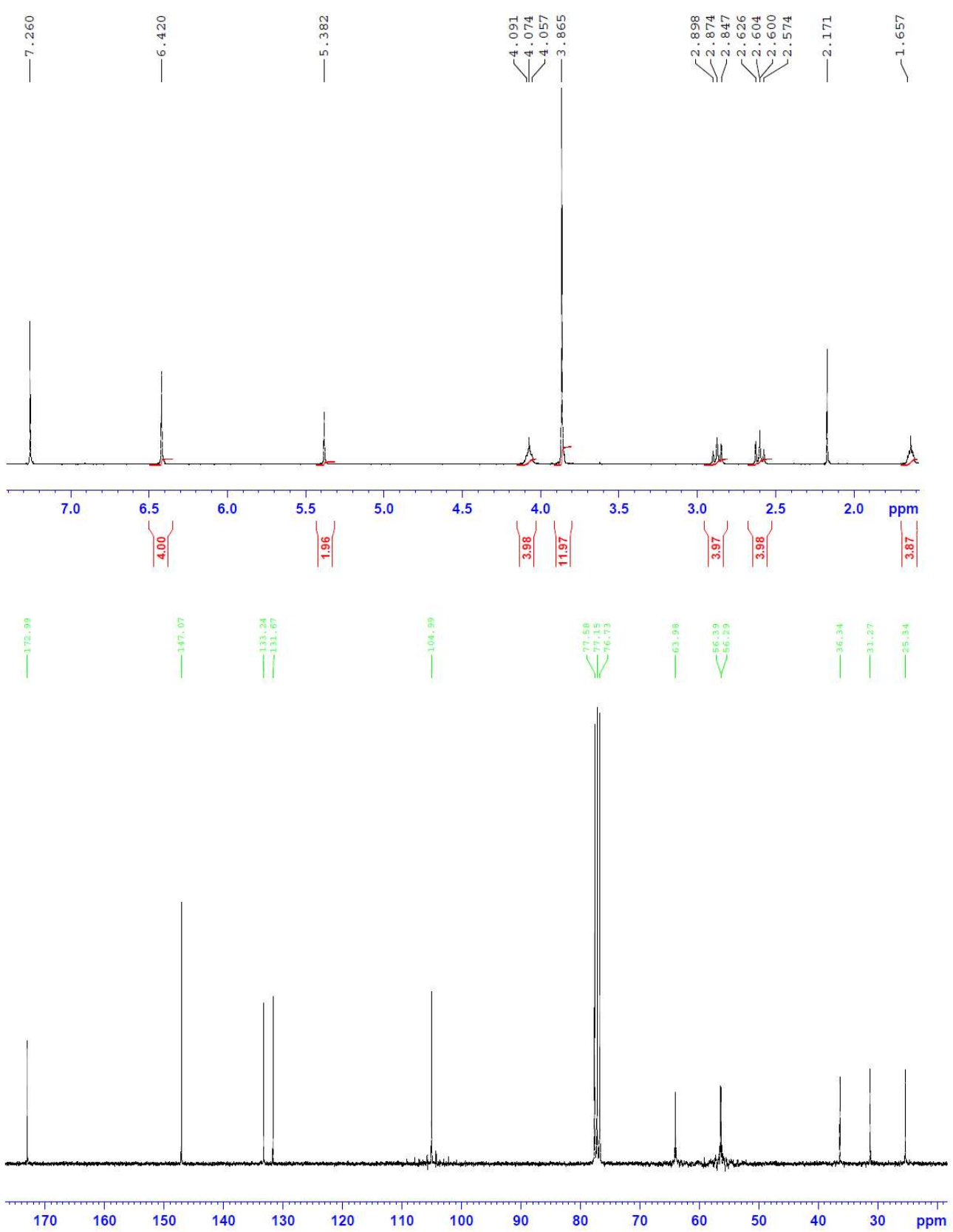


\section{IDS unsat}

White powder (29\%, mp $\left.109^{\circ} \mathrm{C}\right)$. UV: $\lambda_{\max }(\mathrm{EtOH}): 333 \mathrm{~nm}$. FTIR (neat): $v_{\max }: 1701$ (C=O), 2937 (C=C Ar), $3390(-\mathrm{OH}) .{ }^{1} \mathrm{H}$ NMR (300 MHz, $\mathrm{CDCl}_{3}$,

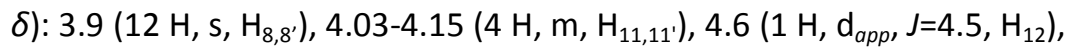
$4.95\left(1 \mathrm{H}, \mathrm{t}_{a p p}, J=4.8, \mathrm{H}_{12^{\prime}}\right), 5.28-5.37\left(2 \mathrm{H}, \mathrm{m}, \mathrm{H}_{10,10^{\prime}}\right), 5.83\left(2 \mathrm{H}, \mathrm{s}, \mathrm{H}_{9,9^{\prime}}\right)$, $6.29\left(1 \mathrm{H}, \mathrm{d}, J=15.9, \mathrm{H}_{2}\right), 6.35\left(1 \mathrm{H}, \mathrm{d}, J=15.9, \mathrm{H}_{2^{\prime}}\right), 6.76\left(2 \mathrm{H}, \mathrm{s}, \mathrm{H}_{5}\right), 6.78$ $\left(2 \mathrm{H}, \mathrm{s}, \mathrm{H}_{5^{\prime}}\right), 7.6\left(1 \mathrm{H}, \mathrm{d}, \mathrm{J}=15.9, \mathrm{H}_{3}\right), 7.65\left(1 \mathrm{H}, \mathrm{d}, \mathrm{J}=15.9, \mathrm{H}_{3^{\prime}}\right) .{ }^{13} \mathrm{C}$ NMR $(75$ $\left.\mathrm{MHz}, \mathrm{CDCl}_{3}, \delta\right): 56.4\left(\mathrm{q}, \mathrm{C}_{8,8^{\prime}}\right), 70.4\left(\mathrm{t}, \mathrm{C}_{11}\right), 73.8\left(\mathrm{t}, \mathrm{C}_{11^{\prime}}\right), 74.1\left(\mathrm{~d}, \mathrm{C}_{10}\right), 78.1$

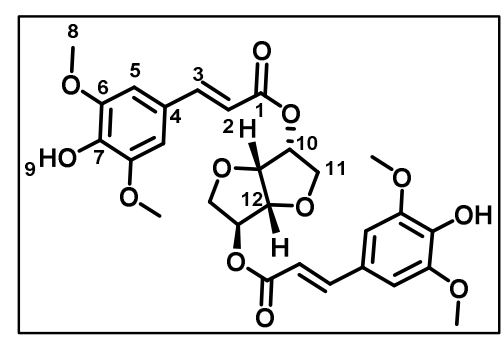
(d, $C_{10^{\prime}}$ ), 81.2 (d, $C_{12}$ ), 86.1 (d, $\left.C_{12^{\prime}}\right), 105.2\left(d, C_{5,5^{\prime}}\right), 114.8\left(d, C_{2,2^{\prime}}\right), 125.7\left(s, C_{4,4^{\prime}}\right), 137.4\left(s, C_{7,7^{\prime}}\right) 146.3\left(d, C_{3,3^{\prime}}\right)$, $147.3\left(\mathrm{~s}, \mathrm{C}_{6,6^{\prime}}\right), 166.2\left(\mathrm{~s}, \mathrm{C}_{1}\right), 166.5\left(\mathrm{~s}, \mathrm{C}_{1^{\prime}}\right)$. HRMS (TOF MS, ES+): $\mathrm{m} / \mathrm{z}$ calcd for $\mathrm{C}_{28} \mathrm{H}_{30} \mathrm{O}_{12} \mathrm{Na}[\mathrm{M}+\mathrm{Na}$ ]: 581.1635; found: 581.1644 .

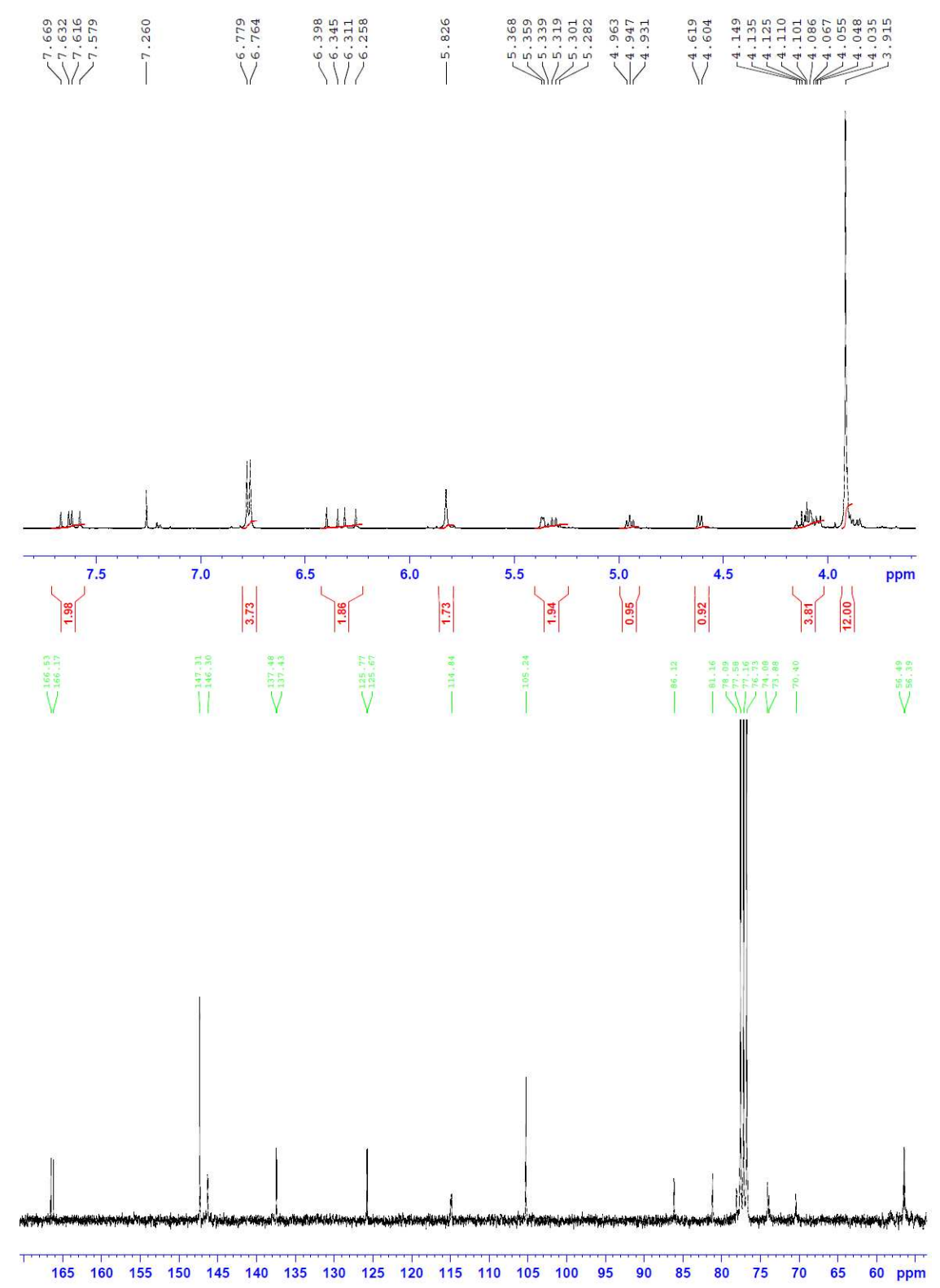




\section{IDS sat}

Yellow oil (30\%). UV: $\lambda_{\max }$ (EtOH): $330 \mathrm{~nm}$. FTIR (neat): $v_{\max }: 1727$ $(\mathrm{C}=0), 2936$ (C=C Ar), $3434(-\mathrm{OH}) .{ }^{1} \mathrm{H}$ NMR $\left(300 \mathrm{MHz}, \mathrm{CDCl}_{3}, \delta\right): 2.68$ $\left(4 \mathrm{H}, \mathrm{m}, \mathrm{H}_{2,2^{\prime}}\right), 2.88\left(4 \mathrm{H}, \mathrm{m}, \mathrm{H}_{3,3^{\prime}}\right), 3.74-3.97\left(1 \mathrm{H}, \mathrm{m}, \mathrm{H}_{11,11^{\prime}}\right), 3.87$ (12 $\left.\mathrm{H}, \mathrm{s}, \mathrm{H}_{8,8^{\prime}}\right), 4.39\left(1 \mathrm{H}, \mathrm{d}, \mathrm{J}=4.5, \mathrm{H}_{12^{\prime}}\right), 4.78\left(1 \mathrm{H}, \mathrm{t}_{a p p}, J=5.1, \mathrm{H}_{12}\right), 5.18$ $\left(2 \mathrm{H}, \mathrm{m}, \mathrm{H}_{10,10^{\prime}}\right), 5.4\left(2 \mathrm{H}, \mathrm{s}, \mathrm{H}_{9,9^{\prime}}\right), 6.42\left(4 \mathrm{H}, \mathrm{s}, \mathrm{H}_{5,5^{\prime}}\right) .{ }^{13} \mathrm{C}$ NMR $(75$

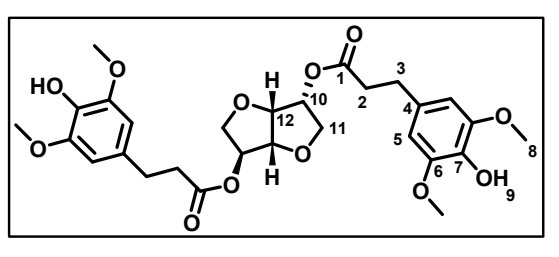
$\mathrm{MHz}, \mathrm{CDCl}_{3}, \delta$ ): $31.0\left(\mathrm{t}, \mathrm{C}_{3,3^{\prime}}\right), 35.9\left(\mathrm{t}, \mathrm{C}_{2,2^{\prime}}\right), 56.2\left(\mathrm{q}, \mathrm{C}_{8,8^{\prime}}\right), 70.3\left(\mathrm{t}, \mathrm{C}_{11}\right), 73.3$ (t, $\left.\mathrm{C}_{11^{\prime}}\right), 73.9$ (d, $\left.\mathrm{C}_{10}\right), 78.0$ (d, $\left.\mathrm{C}_{10^{\prime}}\right)$, $80.7\left(\mathrm{~d}, \mathrm{C}_{12}\right), 85.9\left(\mathrm{~d}, \mathrm{C}_{12^{\prime}}\right), 104.9\left(\mathrm{~d}, \mathrm{C}_{5,5^{\prime}}\right), 131.2\left(\mathrm{~s}, \mathrm{C}_{4,4^{\prime}}\right), 133.2\left(\mathrm{~s}, \mathrm{C}_{7,7^{\prime}}\right), 147.0\left(\mathrm{~s}, \mathrm{C}_{6,6^{\prime}}\right), 172.1\left(\mathrm{~s}, \mathrm{C}_{1,1^{\prime}}\right)$. HRMS (TOF MS, ES+): $\mathrm{m} / \mathrm{z}$ calcd for $\mathrm{C}_{28} \mathrm{H}_{34} \mathrm{O}_{12} \mathrm{Na}$ [M + Na]: 585.1952; found: 585.1948 .

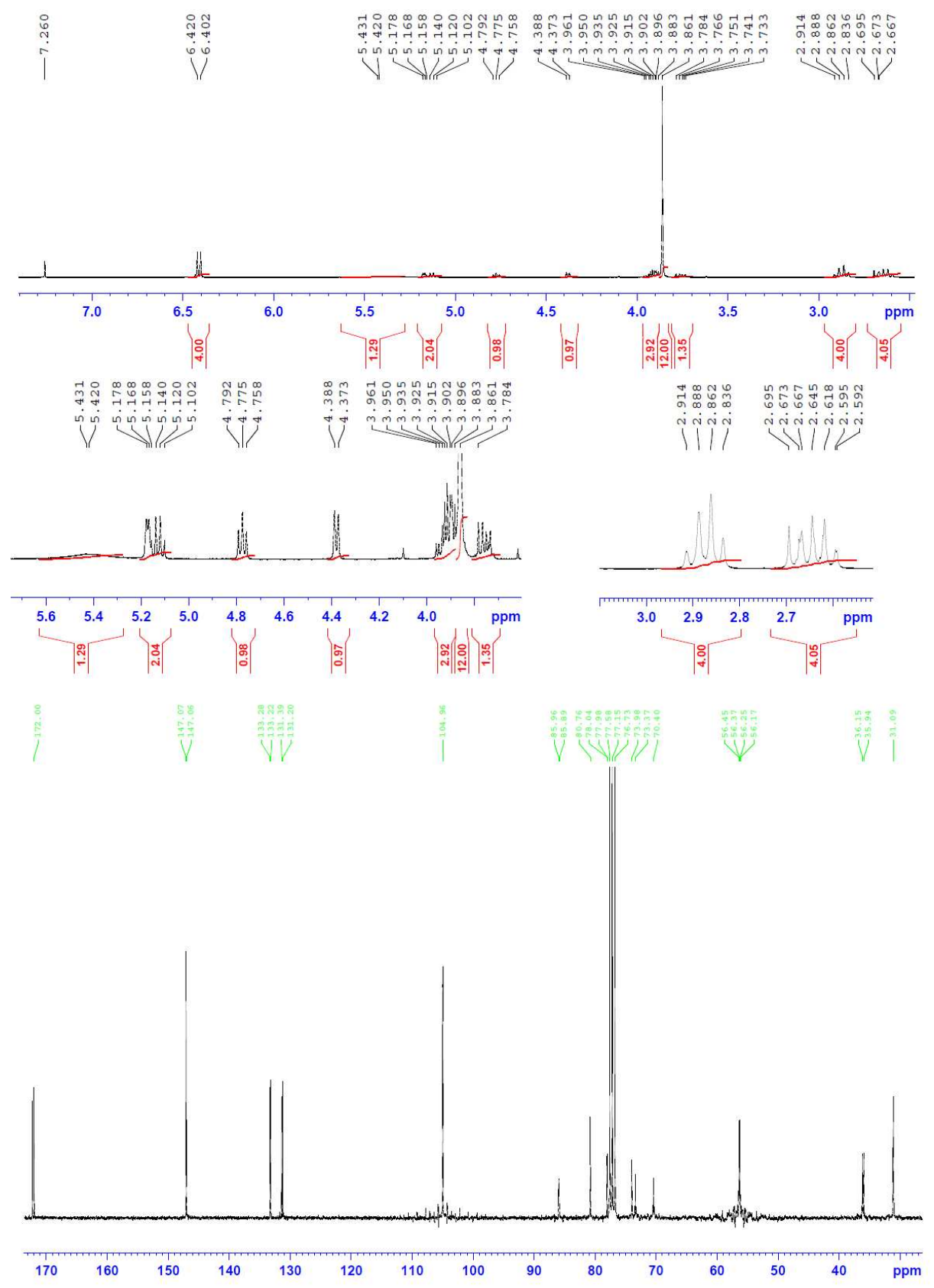


GTF sat

Yellow oil (92\%). UV: $\lambda_{\max }(E t O H): 282,326 \mathrm{~nm}$. FTIR (neat): $v_{\max }$ : 1721 (C=O), 2940 ( $\mathrm{C}=\mathrm{C} \mathrm{Ar}$ ), 3437 (-OH). ${ }^{1} \mathrm{H}$ NMR (300 MHz, $\left.\mathrm{CDCl}_{3}, \delta\right): 2.58\left(6 \mathrm{H}, \mathrm{t}_{\text {app }}, J=8.1, \mathrm{H}_{2,2^{\prime}, 2^{\prime \prime}}\right), 2.85\left(6 \mathrm{H}, \mathrm{t}, J=7.8, \mathrm{H}_{3,3^{\prime}, 3^{\prime \prime}}\right)$,

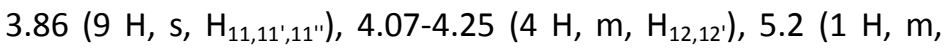

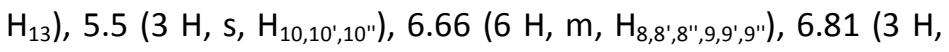

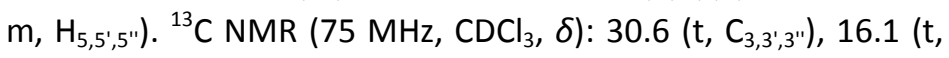

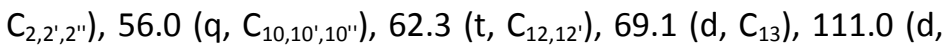

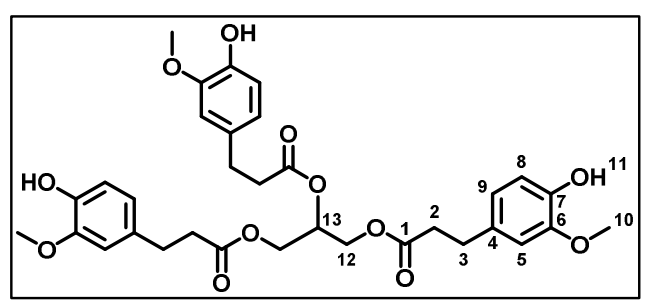
$\left.C_{5,5^{\prime}, 5^{\prime \prime}}\right), 114.5$ (d, $\left.C_{8,8^{\prime}, 8^{\prime \prime}}\right), 120.9$ (d, $\left.C_{9,9^{\prime}, 9^{\prime \prime}}\right), 132.2\left(s, C_{4,4^{\prime}, 4^{\prime \prime}}\right), 144.2\left(s, C_{7,7^{\prime}, 7^{\prime \prime}}\right), 146.6\left(s, C_{6,6^{\prime}, 6^{\prime \prime}}\right), 172.5\left(s, C_{1,1^{\prime}, 1^{\prime \prime}}\right)$. HRMS (TOF MS, ES+): $\mathrm{m} / z$ calcd for $\mathrm{C}_{33} \mathrm{H}_{38} \mathrm{O}_{12} \mathrm{Na}$ [M + Na]: 649.2261; found: 649.2256.
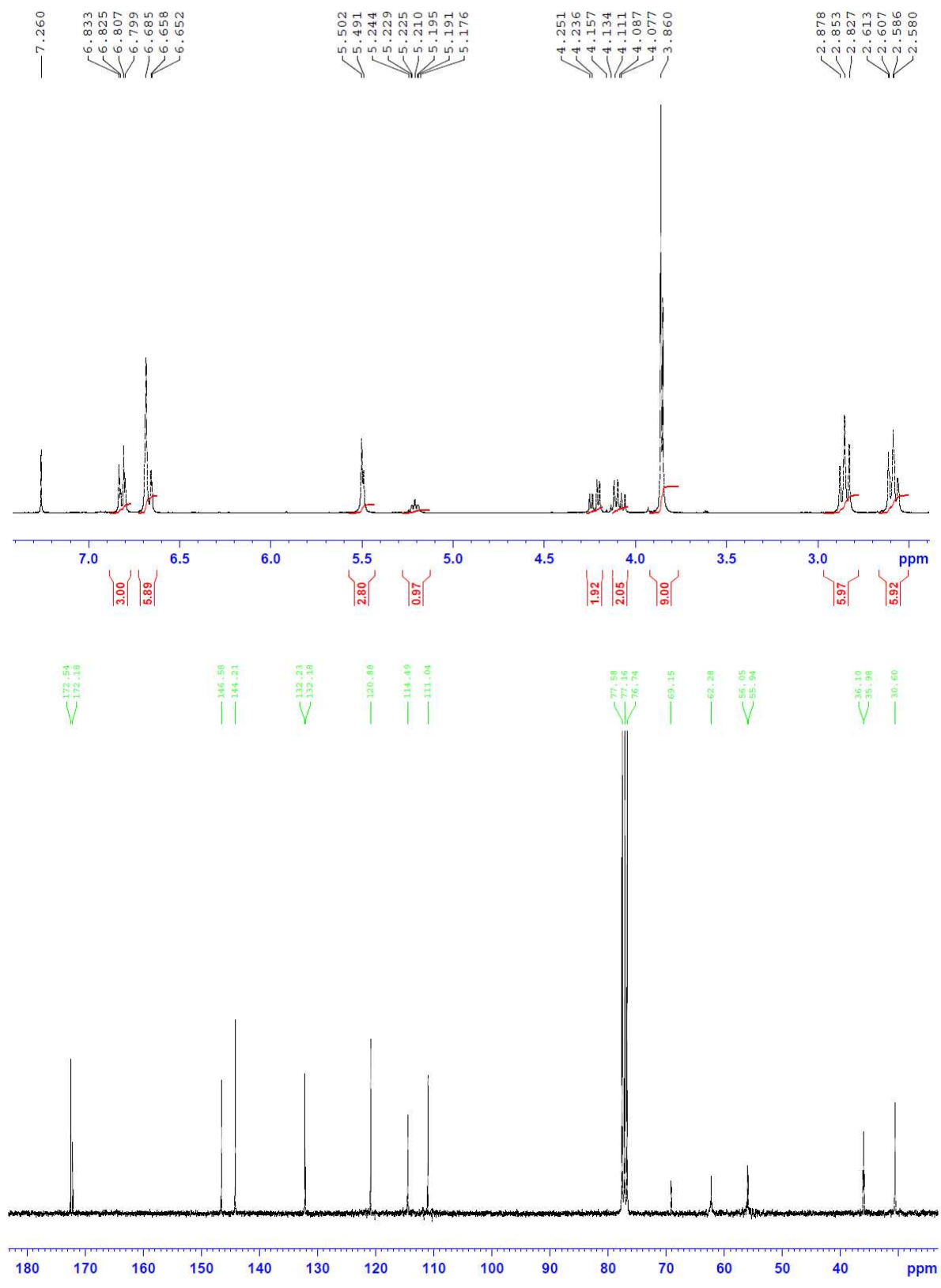

S15 


\section{GTS sat}

Yellow oil (38\%). UV: $\lambda_{\max }(\mathrm{EtOH}): 273 \mathrm{~nm}$. FTIR (neat): $v_{\max }: 1729$ $(\mathrm{C}=\mathrm{O}), 2936$ (C=C Ar), $3450(-\mathrm{OH}) .{ }^{1} \mathrm{H}$ NMR (300 MHz, $\left.\mathrm{CDCl}_{3}, \delta\right)$ : $2.6\left(6 \mathrm{H}, \mathrm{t}, J=8.1 \mathrm{~Hz}, \mathrm{H}_{3,3^{\prime}, 3^{\prime \prime}}\right), 2.85\left(6 \mathrm{H}, \mathrm{t}, J=7.7, \mathrm{H}_{2,2^{\prime}, 2^{\prime \prime}}\right), 3.85$ (18 $\left.\mathrm{H}, \mathrm{s}, \mathrm{H}_{8,8^{\prime}, 8^{\prime \prime}}\right), 4.11\left(2 \mathrm{H}, \mathrm{m}, \mathrm{H}_{10}\right), 4.24\left(2 \mathrm{H}, \mathrm{m}, \mathrm{H}_{10^{\prime}}\right), 5.23(1 \mathrm{H}, \mathrm{m}$, $\left.\mathrm{H}_{11}\right), 5.38\left(3 \mathrm{H}, \mathrm{s}, \mathrm{H}_{9,9^{\prime}, 9^{\prime \prime}}\right), 6.41\left(6 \mathrm{H}, \mathrm{s}, \mathrm{H}_{5,5^{\prime}, 5^{\prime \prime}}\right) .{ }^{13} \mathrm{C} \mathrm{NMR}(75 \mathrm{MHz}$, $\left.\mathrm{CDCl}_{3}, \delta\right): 31.0\left(\mathrm{t}, \mathrm{C}_{3,3^{\prime}, 3^{\prime \prime}}\right), 36.0\left(\mathrm{t}, \mathrm{C}_{2,2^{\prime}, 2^{\prime \prime}}\right), 56.1$ (q, $\left.\mathrm{C}_{8,8^{\prime}, 8^{\prime \prime}}\right), 62.0$ (t, $\mathrm{C}_{10,10^{\prime}}$ ), 69.0 (d, $\mathrm{C}_{11}$ ), 104.9 (d, $\left.\mathrm{C}_{5,5^{\prime}, 5^{\prime \prime \prime}}\right), 131.2$ (s, $\left.\mathrm{C}_{4,4^{\prime}, 4^{\prime \prime}}\right), 133.0$ (s,

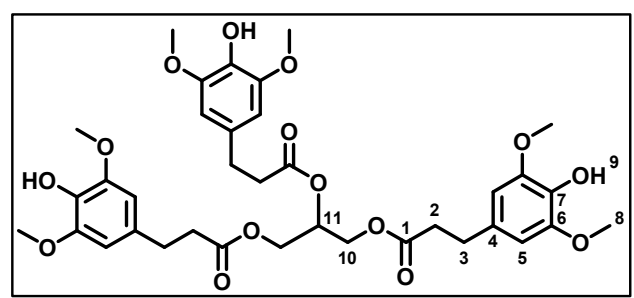
$\left.\mathrm{C}_{7,7^{\prime}, 7^{\prime \prime}}\right), 147.0\left(\mathrm{~s}, \mathrm{C}_{6,6^{\prime}, 6^{\prime \prime}}\right), 172.5$ (s, $\left.\mathrm{C}_{1,1^{\prime}, 1^{\prime \prime}}\right)$. HRMS (TOF MS, ES+): $\mathrm{m} / \mathrm{z}$ calcd for $\mathrm{C}_{36} \mathrm{H}_{44} \mathrm{O}_{15} \mathrm{Na}[\mathrm{M}+\mathrm{Na}]$ : 739.2589; found: 739.2578 .
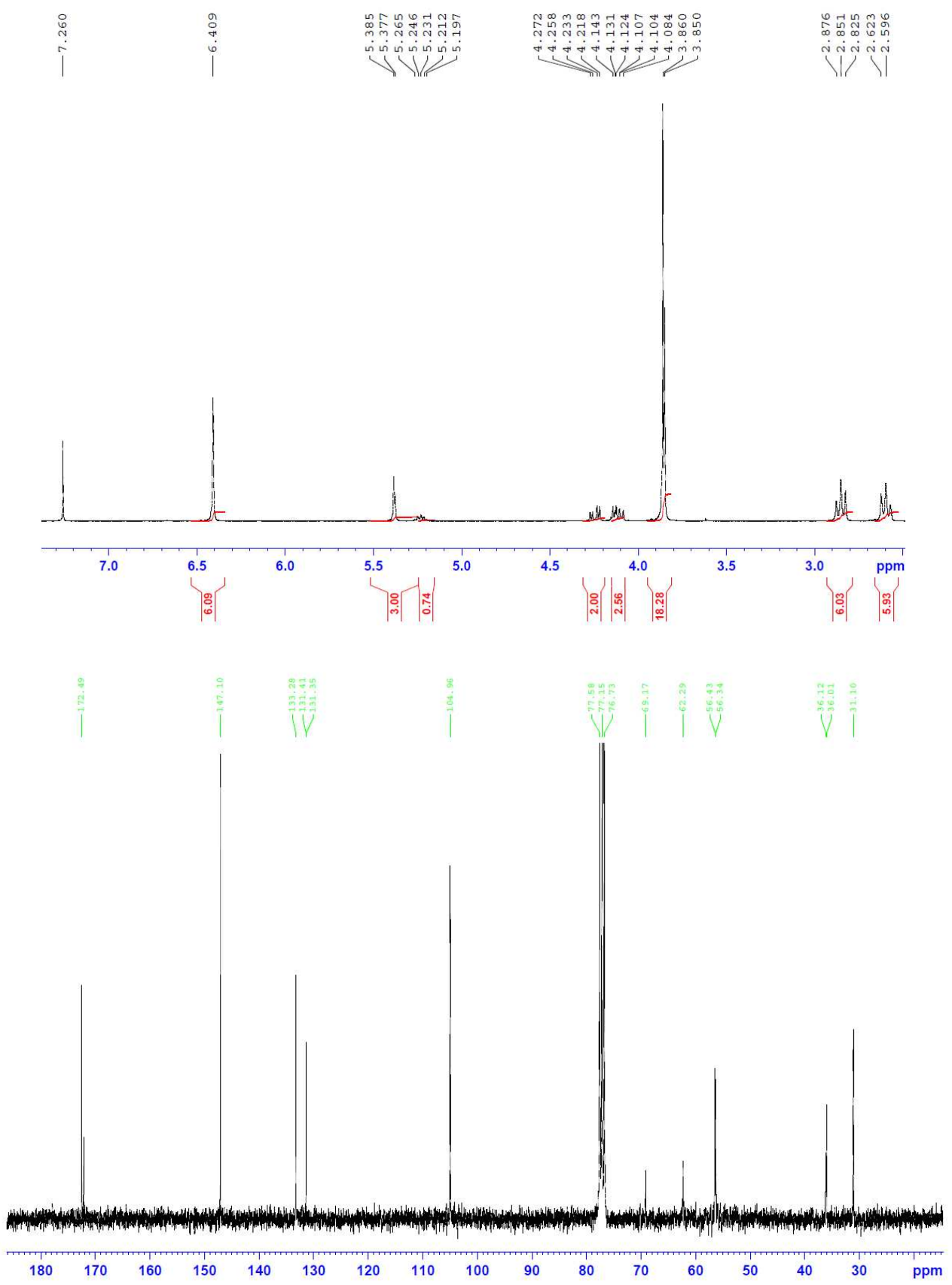